\title{
INDUSTRIA ESPONTÁNEA EN LA PROVINCIA DE ALICANTE
}

\footnotetext{
* Este trabajo es un resumen del informe preparado con el mismo título por encargo del Servicio de Estudios de Presidencia del Gobierno para la elaboración de la Ponencia Española en la reunión intergubernamental de la O.C.D.E. sobre Rural entrepreneurial capacities realizada en Senigallia (Italia) en junio de 1983. El informe constaba de los siguientes apartados:

1) «La población y el poblamiento», realizado por Vicente Gozálvez Pérez. Geógrafo. Universidad de Alicante.

2) «El papel de las estructuras agrarias», por Juan Romero González. Geógrafo. Universidad de Valencia.

3) «La pluriactividad familiar», por Cristóbal Sánchez Cuestas. Sociólogo. Ministerio de Agricultura.

4) «El empresario. Características y cualificación», por José María Tortosa Blasco. Sociólogo. Universidad de Alicante. I.N.E.M.

5) «Estructura de la fuerza de trabajo», por Carmen Lizárraga y José Ramón López Cardón. Economistas.

6) «Características generales de la industria manufacturera», por Miguel Millana Sansaturio. Economista.

7) «Características del sistema espacial de la industria», por Julia Salom Carrasco y Amor Martín Mateo. Geógrafas. Universidad de Valencia.

8) «Especialización dentro del conjunto de las regiones españolas», por Ana Botella Gómez y José María Bernabé Maestre. Geógrafos. Universidad de Valencia.

9) «Especialización en el comercio internacional», por José María Bernabé Maestre. Geógrafo.

Dirección y redacción del resumen, José María Bernabé Maestre.
}

\section{O. RESUMEN Y CONCLUSIONES}

Alicante es una provincia litoral española, tradicionalmente ocupada en una agricultura de secano en clima semiárido de rendimientos muy variables y bajos. En sus extremos norte y sur, sin embargo, entra en contacto con zonas de agricultura regada, con rendimientos elevados y constantes y además con amplias posibilidades de modernización y desarrollo de cultivos comerciales. El valle del Vinalopó ha constituido, además, uno de los pocos pasos por donde se puede acceder de manera relativamente cómoda a las tierras interiores [196] y por ello ha sido zona de paso y de intercambio entre áreas económicas diferentes.

La agricultura tradicional se complementó con un sinnúmero de actividades no agrarias: comercio, transportes, manufactura rural, etc. practicada por los varones e instrumentos agrícolas sobre una base de tiempo parcial, o bien por los elementos familiares con menos dedicación a la tierra (mujeres, niños, etc.). Este hecho permitió densidades de población semejantes a las observables en áreas de regadío vecinas sin apenas signos de miseria rural. La completa utilización de los recursos disponibles y una notable movilidad geográfica y social caracterizaron el área. El acceso a la información era muy bueno para el momento histórico, los centros urbanos modernizaron su estructura comercial y financiera al iniciarse la agricultura comercial y la red de comunicaciones densa.

Cuando a fines del siglo pasado se instrumentalizó una política arancelaria destinada a proteger a la industria local incipiente (del tipo de las denominadas de Industrialización por Sustitución de Importaciones), los empresarios locales encontraron -y aprovecharon- las oportunidades de convertir sus talleres en industrias modernas, especialmente los situados en los centros urbanos mayores y con más tradición manufacturera.

Por último, a partir de 1965, las industrias desarrolladas para suministrar a la demanda interna, encontraron -y también aprovecharon- la oportunidad de dirigirse a los mercados internacionales y competir con las producciones de los países desarrollados, fundamentalmente en base a disponer de unos costes salariales más bajos.

La política de promoción de exportaciones iniciada en 1960 por la Administración 
Pública y la creación de la «Feria Internacional del Calzado e Industrias Afines» por iniciativa de grupos locales fueron elementos muy importantes en el cambio. Con el desarrollo industrial el mercado de mano de obra empezó a regularizarse y legalizarse alcanzando una situación más adecuada durante la década pasada.

Desde este momento, sin embargo, la presión de los costes salariales por una parte, y la competencia de terceros países con costes salariales muy bajos (Taiwán y Corea), están dejando en una situación poco competitiva a la industria local, especializada generalmente en productos poco intensivos en tecnología y de calidades medias y bajas (para los cuales el precio es el factor fundamental de la competencia). Con un mercado comprimido entre los productos de bajo precio de extremo oriente y los productos de moda italianos, y con poca experiencia industrial (la industria apenas tiene quince años), los sectores industriales encuentran dificultades para incrementar la tecnología y el diseño de los productos que ofrecen. [197]

En este momento el paro provincial está alrededor del 20\% de la población activa y en los principales centros industriales puede estar cercano o superar el 30\%. En estas condiciones muchos trabajadores, en proporciones superiores a las tradicionales, han empezado a contratarse por debajo de los mínimos legales y se ha desarrollado una importante economía oculta que está teniendo un efecto disgregador de la estructura industrial. Los trabajadores que quedan en paro encuentran trabajo creando pequeñas empresas cooperativas o contratándose en el mercado «negro».

En una perspectiva de plazo medio el desarrollo de la zona exige promocionar una industria más exigente en tecnología, en diseño, en marketing y que fabrique productos de más calidad. Es necesario además promocionar la diversificación de la estructura industrial, extraordinariamente especializada. Han surgido ya algunas iniciativas: la participación local en la comercialización de los productos está aumentando rápidamente, al igual que la calidad de los productos exportados, la creación de un centro especializado en el control de la calidad de los productos y las materias primas (el INESCOOP, sociedad formada por fabricantes con este fin), y con perspectivas de introducirse en el campo de la creación de tecnología, la promoción de la industria local de maquinaria en los mercados exteriores -al parecer con éxito- por la Cámara de Comercio, etc. Posiblemente el estímulo a estas iniciativas locales y la promoción de otras en esta dirección sean caminos adecuados. A corto plazo, sin embargo, la eliminación del clandestinaje exige estudiar fórmulas de reducción de costes y que se complementen con medidas disciplinarias que hagan rentables las empresas legales hoy existentes.

\section{LAS CONDICIONES PREVIAS}

\subsection{Densidad de población y tamaño de los asentamientos}

Según el Nomenclátor de 1887, se aprecia la existencia de una larga franja litoral de unos $300 \mathrm{Km}$ de longitud por unos 50 de ancho, desde Castellón a Murcia, con elevadas densidades de población y con un sistema de asentamientos bien estructurado y diversificado, donde los núcleos por debajo de los mil habitantes tienen una importancia muy reducida. Hacia el interior la densidad disminuye bruscamente y la dimensión de los asentamientos se reduce: en las montañas los núcleos aunque están próximos físicamente, la fricción de la distancia es muy alta y sus dimensiones son pequeñas. Más allá, en los llanos interiores y en la Meseta de la Mancha, la densidad es muy baja, los asentamientos muy distantes entre sí y de pequeñas dimensiones; están además poco jerarquizados. Un núcleo relativamente grande, Albacete, forma el mercado regional de una gran extensión sin mercados intermedios. [198]

En la medida que el desarrollo ha progresado en la franja litoral se ha producido una corriente migratoria desde las tierras interiores hacia la zona en desarrollo. Esta corriente ha 
reducido la calidad de los asentamientos interiores y su densidad de población en beneficio de la zona litoral, especialmente del gran núcleo urbano de Valencia y su área metropolitana.

Sin embargo, aunque es cierto que la región industrializada alicantina forma parte del área descrita, en su mayor parte ha permanecido como zona rural hasta muy recientemente, aunque haya experimentado un buen crecimiento de la producción, de la población y del empleo.

\subsection{Dotación de recursos}

No parece clara la relación entre recursos e industria local. Para algunos autores la falta de recursos puede ser un factor explicativo del atraso en la industrialización de un área, para otros no es sin embargo relevante. COURTOT explica que en las áreas mediterráneas la diferenciación más relevante en la dotación de recursos aparece entre secanos y regadíos, pues sólo estos últimos tienen posibilidades agrícolas.

La franja litoral no es homogénea desde este punto de vista: abarca secanos y regadíos aunque preferentemente estos últimos. Las amplias llanuras litorales, cubiertas de fértiles aluviones y cruzadas de canales de riego, practican una agricultura intensiva comercializada desde antiguo. En contraste, los áridos valles apenas presentan oportunidades para su población: agricultura pobre, de bajos rendimientos fuertemente controlados por una climatología con gran variabilidad. En estos últimos las hambrunas han sido un fenómeno dramático y recurrente que ha provocado grandes migraciones. La zona de estudio pertenece a este litoral árido y pobre aunque densamente poblado.

Por lo demás, no hay grandes recursos naturales: no existen apenas materias primas industriales, ni minería, etc., aunque no falten pequeñas dotaciones locales: espartizales de Alicante, arcillas de Castellón-Valencia, cáñamo de las huertas, lana de las montañas, etc. Por tanto podrá decirse que aunque el área de estudio sea especialmente pobre en recursos se encuentra en las proximidades de zonas ricas.

\subsection{Las comunicaciones}

Toda la franja litoral es realmente un gran eje de comunicaciones que probablemente era ya importante en época prerromana: la vía «Hercúlea», camino fundamental en las relaciones del litoral mediterráneo. Aún hoy este eje mantiene una gran importancia, remarcada por la autopista del Mediterráneo. La zona de estudio es además una doble zona de paso: por una parte el [199] camino litoral, por otra el camino de penetración hacia las tierras interiores y Madrid. En el valle que estudiamos se solapan el camino litoral con el que conecta el mar con los mercados interiores.

Hemos podido demostrar además que su accesibilidad era ya muy buena antes de la modernización de la agricultura. A fines de siglo era la quinta provincia en el ranking por densidad de caminos. Concluimos por tanto:

a) La zona es un lugar de paso natural bien comunicado desde muy antiguo. No solamente es que la red a escala local es especialmente densa sino que está dentro de uno de los grandes ejes de penetración del principal puerto de Madrid en la primera mitad del siglo XIX. Si aplicamos el esquema de desarrollo de la red de TAAFE, podemos confirmar que las grandes líneas, especialmente por lo que se refiere a las carreteras y caminos reales y al F.C., construidas para el comercio a larga distancia (camino litoral y camino de penetración) se solapan en el área de estudio. Además se constata la existencia de una banda con elevada densidad de comunicaciones que bordea las montañas entre Alicante y Murcia y que penetra por el Valle del Vinalopó hasta Almansa.

b) El Valle comunica zonas económicas aparentemente complementarias. La disposición 
de la red puede entenderse sobre la base de un modelo de interconexión de grandes mercados en tres grandes líneas: a) Comunicación entre las huertas del Segura y Valencia. b) Comunicación entre el puerto de Alicante y los mercados interiores (Albacete y Madrid). c) Comunicación entre los núcleos textiles de la montaña y sus áreas de suministro de materias primas y de mercado de los productos terminados (Andalucía y la Mancha).

Se considera que este factor permitió a la zona de estudio unas relaciones más amplias para sus habitantes y, por tanto, un horizonte geográfico y de conocimientos mucho más amplio y consecuentemente una mentalidad más abierta a nuevas ideas. Además, este recurso proporcionó la posibilidad de comercializar parte de la producción agrícola o los productos transformados por las comunidades locales y la participación en este flujo permitió una experiencia de gran valor.

\subsection{La propiedad de la tierra}

La tierra del valle está en manos de pequeños propietarios en su mayor parte. También ocurre lo mismo con la mayor parte de la extensión cultivada de la franja litoral, como consecuencia de un original proceso de desamortización y de liberación de las cargas feudales. El profesor Gil Olcina ha explicado cómo el régimen señorial de gran parte de las tierras valencianas ha conducido, a través del mecanismo de la enfiteusis, a la pequeña propiedad rural. Pero aún antes de que el campesino adquiriese la plena propiedad de la [200] tierra la enfiteusis le proporcionaba la dirección de la empresa agraria a cambio de un canon.

La pequeña propiedad proporcionó al agricultor una experiencia de gestión de los cultivos, una posibilidad de promocionarse a través del propio esfuerzo que quizá no han dispuesto las áreas con otras formas de propiedad.

Es difícil saber hasta qué punto es cierta la afirmación. Si bien la zona de estudio se ha caracterizado por la pequeña propiedad, ha habido importantes diferencias de unos municipios a otros. La zona de Elche ha tenido grandes propiedades señoriales, al igual que Almansa. Villena es más parecida en su estructura de la propiedad a los pueblos castellanos que a los valencianos. Por otra parte, muchos municipios de la Comunidad Valenciana han dispuesto de pequeñas propiedades sin por eso conseguir ni tan siquiera modernizar su agricultura.

Puede decirse que aunque la pequeña propiedad es un factor importante, cuentan otros como el aislamiento y la dotación de servicios del área. Sin buenas comunicaciones y sin asentamientos con servicios mínimos puede ocurrir que perdure la agricultura de subsistencia, emigre la población y termine por la desertización del área. Con buenas comunicaciones, accesibilidad, información y núcleos bien comunicados y de dimensiones suficientes se puede alcanzar el camino del desarrollo.

\subsection{Utilización de la fuerza de trabajo}

Nuestro punto de vista es que la utilización intensiva de la fuerza de trabajo en la comunidad campesina permite elevadas densidades de población en ambientes con baja dotación de recursos y que en estas condiciones las comunidades son especialmente sensibles a las oportunidades de desarrollo industrial.

Según el censo de 1860, nuestra zona de estudio se caracteriza por un elevado porcentaje de población dedicado a la agricultura (70-80\%) en contraste con las áreas del interior (50-60\%). Esta diferencia es compensada por un mayor porcentaje en el sector terciario, especialmente en criados que en las zonas interiores alcanzan porcentajes de hasta un $20 \%$, y de pobres $(6-7 \%$ en el interior, $2 \%$ en la zona de estudio). Los resultados son por tanto aplastantes: en las zonas interiores con una utilización extensiva de recursos y propiedad de grandes dimensiones, no se 
consigue ocupar totalmente a la mano de obra disponible y se produce una elevada polarización de las rentas. Lógicamente el porcentaje de propietarios es mucho mayor en la zona de estudio que en el interior. En la zona de estudio se combina una densidad de población enormemente mayor, un suelo más pobre, una distribución de rentas más equilibrada y menor pobreza de las gentes. [201]

Es necesario sin embargo explicar cómo pueden vivir tantos campesinos con tan poca tierra. En todo el valle las relaciones escritas hacia estas fechas (Cavanilles, Madoz, etc.) citan con frecuencia la existencia de trabajos complementarios que ocupan normalmente mujeres, jornaleros en épocas de escaso trabajo estacional, etc. Este tipo de información no es reflejada desgraciadamente en los censos de población, por lo que no puede cuantificarse, aun cuando den cuenta de su importancia los estudiosos del momento. Este tipo de trabajos implica la pluriactividad de la familia campesina que estudiamos en el siguiente párrafo.

\subsection{La pluriactividad familiar}

Nuestro punto de vista es que la pluriactividad favorece la industrialización del medio rural. Esto es así porque conecta a la familia campesina con actividades no agrarias de manera paulatina a través, por ejemplo, de aumentar el peso de la manufactura y los servicios en la ocupación familiar y rompe los esquemas rígidos de los papeles familiares mediante la incorporación de los miembros «tradicionalmente inactivos» en activos -fundamentalmente mujeres-, lo que implica una cierta flexibilidad en la organización del trabajo familiar.

La pluriactividad supone, por un lado, la utilización más intensiva de los recursos disponibles con ocupación de la mano de obra, especialmente del subempleo o paro encubierto porque hay puestos de trabajo o, al menos, las condiciones de iniciativa local para crearlos. Además, exige, y propicia a la vez, un cierto nivel de equipamiento que permita el desarrollo de la actividad.

Lo difícil es medir este fenómeno en una economía tradicional; sólo por medio de contrastes muy minuciosos entre distintas fuentes en el municipio de Elda hemos empezado a entrever la enorme importancia de este fenómeno. En los momentos de menos trabajo los jornaleros y pequeños agricultores arrancaban el esparto de las montañas y lo majaban, seguidamente las mujeres de los mismos grupos sociales lo trenzaban para formar esteras que eran comercializadas en los mercados de las huertas y del interior. Una gran parte de los pequeños agricultores e incluso jornaleros realizaban una o dos veces al año un viaje a distancias largas como trajineros, buhoneros, arrieros, etc. y de esta forma ocupaban el momento de paro estacional tanto para ellos como para sus caballerías. Otros emigraban estacionalmente a segar el trigo a la Mancha y Aragón o el arroz a la Ribera, o a trabajar en sus oficios. La exigencia de mano de obra para el majado en el momento punta de la ocupación la resolvieron adaptando un martinete de majar a los molinos harineros de la zona. Además, la mayor parte de los artesanos son además agricultores. Sólo un pequeño grupo de propietarios de la tierra mantienen elevados niveles [202] de desocupación de recursos y contratan a criados para que mantengan los trabajos de la casa, actividad que pasa así a ocupar más del doble de personas que en el resto de familias a pesar de que tienen menos hijos menores.

a) En las familias de jornaleros y pequeños propietarios la pluriactividad familiar es muy elevada, pero también especializada en algunos campos: los hombres trabajan por cuenta ajena en la tierra, recogen el esparto para elaborar felpudos o realizan transporte a pequeña escala con asnos; las mujeres realizan artesanías del esparto.

b) En los propietarios medios se entrecruza la explotación de la tierra con la menestralía. La pluriactividad y el trabajo a tiempo parcial son los rasgos dominantes. La movilidad social y los cambios de oficio son relativamente frecuentes en este grupo, aun cuando este aspecto no 
lo tengamos cuantificado.

c) En las familias de propietarios mayores, la tasa de actividad es baja, pues no trabajan las esposas y raramente los hijos, alguno de los cuales está censado como estudiante. En el trabajo doméstico colabora casi una criada por casa. La interpenetración con oficios que con el tiempo pueden convertirse en industriales es prácticamente nula, pero sin embargo es el grupo que genera las mayores acumulaciones de capital.

La relación entre estas actividades transformadoras difusas en el medio rural y la industria posterior ha sido reconocida por muchos autores (Anderson, 1982). En la zona de estudio casi la totalidad de las industrias hoy existentes tienen su origen en estas antiguas actividades, pues los municipios mantienen las mismas especialidades.

Por tanto, puede afirmarse que en la zona posteriormente desarrollada el grado de ocupación de la mano de obra y de los recursos era mucho más elevado que en el resto. Primero porque se ocupaba un porcentaje mayor de ambos factores y segundo porque se hacía de una manera más intensa al cubrir con trabajos complementarios las épocas muertas. Este fenómeno explica por qué elárea de estudio podrá mantener elevadas densidades de población basadas en una agricultura árida de bajos rendimientos. Al mismo tiempo estos trabajos complementarios son el lazo que conecta la agricultura tradicional con la industria moderna, como se demuestra por el hecho de que las especialidades actuales sean las mismas que las de la antigua manufactura dispersa.

Por tanto, la industria surge en un ambiente caracterizado por un exceso de población frente a los recursos disponibles, en una zona bien comunicada, con una densa estructura de asentamientos donde además existe una antigua tradición transformadora. Todo ello aprovechando la experiencia de gestión y [203] las pequeñas acumulaciones que la agricultura formó en manos de pequeños propietarios.

Esta discusión delimita algunas cuestiones respecto de las condiciones previas necesarias para desarrollar este tipo de industria. El efecto de la demanda de la agricultura sobre las actividades transformadoras ha jugado papeles diferentes según zonas. Para la zona de estudio la demanda estrictamente local ha sido poco importante, aunque los mercados para sus productos no hayan estado demasiado lejos. En la industrialización de las zonas de huerta la demanda local ha sido fundamental y de ahí han nacido dos estructuras productivas distintas, al menos en lo que se refiere a la industria: municipios con una estructura industrial altamente especializada en los secanos y municipios relativamente diversificados en las zonas de regadío. Además los municipios de las zonas de regadío presentan especialidades que tienen justificación desde el punto de vista de la teoría de la localización, mientras que las de los pueblos de secano sólo pueden justificarse por la existencia de iniciativa local.

\subsection{La modernización de la agricultura}

Según el modelo de Fei y Ranis la economía subdesarrollada se compone de dos sectores: agrícola o tradicional e industrial urbano. La modernización de la agricultura provoca una caída de los salarios en áreas rurales y la emigración de la mano de obra a las ciudades. Esta corriente migratoria alimenta el empleo industrial. Por tanto, modernización (mecanización) de la agricultura, industrialización y urbanización son fenómenos paralelos. Además la agricultura puede ser también la fuente de capital para la industria, aun cuando debemos precisar diferencias según el tipo de capital: para los primeros desarrollos industriales el equipo previamente creado (molinos, medios de transporte, edificaciones) pueden ser puntos de partida clave; también la utilización de infraestructuras previamente creadas (vías de comunicación, p. e.); también se suele indicar que es importante la transferencia de capital líquido generado en la agricultura o en la comercialización de la producción agrícola a la industria o a la comercialización de la 
producción agrícola.

Un último punto, no tan citado, es que el desarrollo de una agricultura comercial induce un desarrollo de los servicios urbanos (financieros, agencias de transporte, etc.) y desarrolla una infraestructura viaria, crea un flujo de información desde zonas lejanas y, por último, bajo ciertas condiciones de propiedad de la tierra, genera una experiencia de gestión muy difundida entre los empresarios agrícolas.

En la zona de estudio el desarrollo de la agricultura comercial es relativamente temprano, pues desde mediados del siglo XVIII puede constatarse la [204] extensión de los viñedos. Tuvo su punto máximo en la segunda mitad del siglo XVIII y posteriormente decayó sin posibilidad de sustitución al ponerse en cultivo áreas más productivas aunque quizá inicialmente menos accesibles. Durante esta época los cultivos comerciales se extendieron por toda la franja litoral y los puertos que hasta este momento eran básicamente importadores incrementan enormemente el volumen de su comercio y se convierten en exportadores. Bajo las tecnologías primitivas de transporte de la época los cultivos comerciales no podían extenderse mucho hacia el interior.

¿Qué cambios se producen inducidos por este fenómeno? La red de comunicaciones era ya buena, como hemos explicado, entre otras razones porque se beneficiaba del F.C., creado en 1859 para conectar el puerto de Alicante con el principal mercado del interior (Madrid). Pero con la extensión de los cultivos comerciales se incrementan de modo muy notable las vías de comunicación, de modo que la provincia pasa a ocupar el quinto lugar en el ranking de las provincias españolas por la densidad de carreteras (a continuación de los grandes centros de desarrollo). Esto no sólo afecta a la modernización de las grandes vías del comercio a larga distancia sino, sobre todo, a las carreteras comarcales que conectan los núcleos rurales entre sí o con los grandes ejes de comunicación, promocionando en los enlaces centros comerciales intermedios.

En cuanto a la estructura comercial, la intensa comercialización de productos agrícolas y la agricultura desarrollada para ello, produjeron la desaparición de la economía de autoconsumo y la demanda generada por estas actividades de servicios comerciales se multiplicó. El sistema de comercio tradicional está basado en los mercados periódicos y mercaderes itinerantes, debido a la débil demanda de las zonas rurales; el moderno implica establecimientos fijos, para satisfacer una demanda mucho mayor y más difuminada en el tiempo. En los centros rurales, y especialmente en los intermedios, aumentan notablemente las tiendas minoristas y los establecimientos y almacenes mayoristas, estos últimos con la función adicional de financiar al agricultor. Al calor de esta demanda se desarrollan también industrias que atienden a la demanda local (textiles, etc.) o que elaboran materias primas locales (jabón, aguardiente, etc.) o fabrican y reparan sencillos equipos (maquinaria agrícola), lo que convierte a estos centros intermedios en industriales (aunque no especializados). También aquí se desarrollan los primeros servicios financieros: en primer lugar por el papel jugado por los comerciantes mayoristas en la financiación y en segundo porque es aquí donde se instalan las primeras sucursales bancarias y donde se crean las primeras Cajas de Ahorros. Los beneficios obtenidos por comerciantes o agricultores locales pasan a aplicarse al comercio mayorista (bodegueros y comerciantes de vinos) y posteriormente al negocio financiero (usureros, banqueros). Además las oficinas de las grandes [205] casas comerciales francesas ejercen un papel semejante. Por último, estos centros urbanos también se convierten en centros de difusión de innovaciones, pues aquí se instalan agencias y servicios que proporcionan información sobre la situación de los mercados, la tecnología, etc.

Sin embargo, las primeras ciudades industriales, aun cuando utilizaron estas infraestructuras, no siempre coinciden con los centros comerciales agrarios, antes citados. Los centros comerciales de la agricultura no siguieron el camino de la industrialización: posiblemente porque su especialización en la comercialización de productos agrícolas y la desconexión de los 
mercados industriales regionales les dejaron en inferioridad con respecto a las zonas vecinas; pero sobre todo porque al estar situados sobre los mejores suelos de la zona y con más posibilidades de riego, el proceso de modernización de la agricultura no quedó paralizado a partir de la crisis del vino.

En conclusión, podemos afirmar que el desarrollo de la agricultura comercial no produjo un flujo migratorio desde las áreas rurales a las urbanas, más bien al contrario, la modernización de la agricultura redujo las corrientes migratorias establecidas a mediados del siglo. Por tanto, el incremento de la agricultura produce un incremento generalizado de la población e incluso una dispersión de los efectivos. Además, aun cuando la demanda local estimula el desarrollo de un conjunto de industrias, sólo sobreviven a la crisis agrícola aquéllas que han alcanzado los mercados nacionales, que precisamente son las herederas de la antigua manufactura dispersa. En las primeras etapas el capital líquido generado en la agricultura es transferido a la industria, por inversión directa o a través del préstamo de usureros y banqueros formados en el comercio de productos agrícolas. Sin embargo, en la medida que se crean sucursales de los grandes bancos, la fricción de la distancia pierde sentido para el dinero líquido y consecuentemente se corta la transferencia de capital agricultura-industria a través de estos mecanismos. La inversión directa de grandes propietarios o comerciantes en la industria fue muy rara y no conocemos ningún caso en que tuviera éxito; puede decirse que los comerciantes ricos prestaron sus fondos, mientras que los arruinados en el proceso de crisis del vino intentaron la vía industrial.

\section{LOS PROCESOS}

El proceso de desarrollo del área en cuestión tiene características que lo asemejan al de otras áreas. Como se ha señalado repetidamente, el desarrollo reciente de estas áreas está unido a la demanda exterior de los productos industriales fabricados por ellas; este factor adquiere importancia después de la segunda guerra mundial por la mejora en la red de comunicaciones, el aumento de costes y de la inelasticidad, onerosidad e ingobernabilidad de la [206] estructura industrial de los países desarrollados para satisfacer su propia demanda interna de productos cada vez más diferenciados. Estos productos, que exigen mucha mano de obra y trabajan con tecnologías no muy complejas, proporcionan una oportunidad a las regiones no desarrolladas, aunque con determinadas precondiciones. Algunos autores señalan la oportunidad de las políticas orientadas a la exportación para estos países. Aunque estas políticas hayan mostrado un elevado nivel de eficiencia, para que puedan ser puestas en práctica es necesario que el país tenga unos niveles de desarrollo previos. Estos niveles mínimos se refieren a la capacidad de gestión y a la práctica en el uso de tecnologías. Por ello muchos autores opinan (Chenery, Lee) que antes de que se produzca el desarrollo de industrias orientadas a la exportación es necesario que haya una mínima base industrial, generalmente creada a través de políticas de industrialización por sustitución de exportaciones. Por tanto, antes de que se inicie la exportación, hay una larga historia a través de la cual se ha creado la cualificación profesional, la capacidad de gestión y las infraestructuras necesarias que condicionan el nivel de participación local e incluso las especialidades de la producción.

Otro aspecto importante se refiere a la manera como se distribuyen los ingresos en este tipo de regiones en desarrollo. Se ha dicho que a este respecto se puede diferenciar dos tipos: con marginación y con equidad. El modelo con equidad se caracteriza por una distribución igualitaria de los ingresos tanto entre grupos sociales como entre los distintos espacios, lo cual induciría corrientes migratorias reducidas. Es, por tanto, un desarrollo difuso y relativamente igualitario, con un elevado grado de participación. Parece que este fenómeno se produce cuando la igualdad de oportunidades era elevada antes de la industrialización, la polarización de rentas escasa, la información relativamente distribuida y la participación en el proceso de industrialización fue 
grande.

En resumen, podemos detectar tres aspectos clave en este modelo de industrialización: elevado porcentaje de la producción orientado a la exportación, una larga historia industrial y de gestión antes del inicio de las exportaciones, que posibilita la participación de la iniciativa local y el carácter equitativo de la distribución de las rentas generadas por la industrialización.

\subsection{Difusión de innovaciones}

Otro aspecto importante es el acceso a la información: sobre procesos de producción, mercados, financiación, etc. La introducción de nuevas actividades en el ambiente rural implica un cambio más o menos importante y todo cambio implica un riesgo, que se reduce mejorando la información de los tomadores de decisiones. El riesgo asumible por el campesino depende de su [207] experiencia en la toma de decisiones y del capital disponible sin comprometer la supervivencia de la familia. Por ello hay un encadenamiento entre viejas y nuevas técnicas. Si la tierra permite aumentar su rentabilidad, el campesino tiende a mejorarla (como ocurre en las zonas de regadío), en caso contrario se desplaza hacia otro tipo de actividades sobre las cuales dispone de una cierta información: porque las conoce a través de la pluriactividad familiar o porque conoce sus mercados y la tendencia actual de la demanda a través del mismo mecanismo y su técnica de fabricación es fácil.

El acceso a la información en la zona posteriormente industrializada y en sus vecinas ha podido medirse a mediados del siglo pasado, y según esta operación ya entonces estaba mucho mejor informada que las zonas circundantes. Además, las condiciones que hemos visto de fuerte movilidad social y geográfica de sus gentes ha posibilitado un drenaje de conocimientos muy importante en el desarrollo industrial. Lo más notable es, sin embargo, la diferencia entre el secano bien comunicado y con buena cantidad de información (medida por el servicio de correos) que se industrializó durante el siglo y medio siguiente, y las tierras de secano aisladas del interior que han terminado en una despoblación casi absoluta.

En el proceso de modernización de las áreas rurales, al menos lo hemos visto en la introducción de actividades industriales, muchas veces puede distinguirse entre los primeros introductores de una innovación (una nueva técnica de producción, un nuevo mercado, etc.) y los que imitan el procedimiento una vez que se ha demostrado rentable. En el primer grupo puede haber gentes de todo tipo (comerciantes, artesanos, molineros, fracasados de la agricultura, etc.), pero con cierta frecuencia nos encontramos con emigrantes que vuelven a su lugar de origen con algo de capital y conocimientos sobre procesos de producción y mercados que intentan utilizar montando pequeñas instalaciones en sus pueblos (el emigrante que vuelve de una ciudad industrial trae conocimientos, ahorros y ciertas relaciones personales de gran utilidad en su lugar de origen). La imitación se produce a través de relaciones familiares y de trabajo. En algunos casos las relaciones familiares presumiblemente tienen mucha importancia porque, además de servir de canal para la transmisión de la información, sirve de apoyo logístico para las nuevas empresas -especialmente como garantía financiera-. Las relaciones de trabajo son sin embargo el aprendizaje de los nuevos empresarios en la mayor parte de los casos, dentro de ellas los empleos de gestión, administración y ventas son los que parecen proporcionar los mejores empresarios. Esto -junto con otros datos estudiados- nos permite suponer que junto a la información es necesario un cierto nivel de cualificación cuando la empresa supera el ámbito local o comarcal: saber leer y escribir bien, algo de experiencia administrativa y una cierta cualificación (elementos de contabilidad y cálculo). No sólo es que la [208] zona posteriormente industrializada muestra desde mediados del siglo pasado unos niveles de alfabetización más altos que sus vecinos, sino que también las familias que dieron origen a las iniciativas de actividades industriales pueden diferenciarse de las que no (aunque tuvieran el mismo nivel de 
conocimientos, p.e. como miembros del mismo grupo de artesanos) por su nivel de alfabetización. La mayor parte de estos conocimientos en su vertiente técnica (contabilidad, cálculo, correspondencia, etc.) se difundieron a través de canales diferentes de la enseñanza formal pública. Los hijos de campesinos, artesanos, etc. asistían a pequeñas academias rurales o urbanas privadas, donde aprendieron los rudimentos para su cualificación como futuros empresarios.

La difusión, sin embargo, no siempre se produjo de manera espontánea. Brown ha indicado que aparte del enfoque tradicional que considera la difusión de la innovación como un proceso de aprendizaje (perspectiva de adopción), puede considerarse otro tipo de enfoque que él denomina de infraestructura y mercado. El enfoque parte de la idea de que muchas veces existe una política consciente de difusión de la información, de oferta de información. Desde este punto de vista pueden distinguirse tres tipos de actividades: instalación de agencias de difusión, puesta en práctica de estrategias en las agencias para inducir la adopción de innovaciones y la adopción misma. Las empresas constructoras de maquinaria y las comerciales de materias primas han jugado un papel importante en este proceso de difusión en las primeras etapas: facilitaron viajes de empresarios seleccionados a los países de origen para que pudieran ver empresas funcionando con las nuevas tecnologías y/o montaron empresas en las zonas zapateras promocionando la nueva tecnología a través de visitas. La actuación pública en este sentido sólo ha tenido importancia muy recientemente a través de determinadas iniciativas generalmente inducidas por hombres especialmente sensibilizados de las zonas industrializadas: Feria Internacional del Calzado, Inescoop. Estas actuaciones han consistido en crear agencias de información que han tenido mucha importancia en la relación con los mercados lejanos para conocer las nuevas tecnologías de producción, etc.

La difusión como aprendizaje por la acción ha sido crucial en las primeras etapas, y como consecuencia los núcleos rurales terminaron especializándose en la actividad que los primeros empresarios con éxito llevaron a cabo. Posteriormente a través de las relaciones de trabajo, las primeras especialidades saltaron a los núcleos vecinos dando lugar a paisajes industriales en mancha de aceite con especializaciones muy características. A través de una encuesta entre industriales sabemos que mucha información se transmite a través de relaciones personales y profesionales entre clientes y proveedores y entre contratista y subcontratista. Las agencias de información más importantes son las ferias internacionales y las delegaciones de las empresas de maquinaria [209]. Una parte de la información, sin embargo, es transferida a través de revistas especializadas y propaganda impresa.

En resumen: Determinados rasgos del paisaje industrial permiten afirmar que el acceso a la información ha sido un aspecto crucial en el desarrollo de actividades industriales en base a iniciativas locales. El retorno de emigrantes ha sido una fuente importante en producir innovadores. El nivel cultural, la movilidad geográfica y la pluriactividad familiar parece que han sido elementos importantes para facilitar la adopción de innovaciones. La difusión de innovaciones se ha producido a través de relaciones familiares y de trabajo, la enseñanza formal ha tenido poca importancia, aunque seguramente porque no ha sabido adaptarse a las necesidades locales (sólo la enseñanza privada ha sabido hacerlo en algún caso). Las agencias de difusión más eficientes han sido las delegaciones de las empresas de maquinaria y las Ferias, aunque posiblemente porque no se ha llevado a cabo ninguna otra actuación pública. Los pocos intentos de crear agencias de difusión se han encontrado con graves problemas de falta de demanda para los servicios ofertados, posiblemente por falta de adecuación. En estos ambientes rurales con recursos subutilizados (sobre todo humanos) y cierta sobrevaloración del riesgo, los efectos de imitación o «bandwagon» tienen mucha importancia, determinando muchas veces las especializaciones locales. 


\subsection{Crecimiento del empleo}

El origen de estas industrias rurales arranca del ambiente de subempleo y emigración de la segunda mitad del siglo pasado. Desde este momento se puede constatar una corriente migratoria que sale hacia el norte de África, Francia y, a finales de siglo, hacia América (Brasil). Muchos son trabajadores del campo que marchan en busca de trabajo estacional, de manera semejante a como lo hacían hacia Castilla y Huerta de Valencia, pero en parte son pequeños propietarios o hijos de agricultores que van a colonizar las tierras nuevas. La zona, como hemos explicado anteriormente, tiene una densidad de población muy alta para los recursos agrícolas disponibles y, a pesar de la pluriactividad, se enfrenta con importantes excedentes demográficos. En los momentos de crisis de la agricultura o de grandes hambrunas provocadas por las sequías, la tierra no es capaz de mantener a la población ocupada más que en una mínima parte y consecuentemente se producen grandes oleadas de emigración.

Aun con una propiedad de la tierra relativamente distribuida y con una modernización temprana de las explotaciones, la tierra de secano no puede dar ocupación a toda la población demostrando que sólo la industria puede evitar la emigración. [210]

Los productos elaborados en manufactura rural compiten bien con los de los artesanos urbanos (a pesar de la desaparición de la institución gremial), en parte porque el campesino está dispuesto a hacer el trabajo con menos ingresos pero sobre todo porque no espera obtener de aquí todos los recursos para la vida de la familia y porque aplica a este trabajo todos los miembros no utilizados por la agricultura. La pluriactividad familiar es aparentemente la razón por la que los centros rurales compiten en los centros de consumo urbanos con la artesanía local.

A partir del desarrollo industrial en los principales centros, la emigración se va paralizando hasta convertirse en inmigración. Los principales (Elda, Elche, Alicante) están atrayendo población desde 1910, primero de municipios cercanos y después de los alejados, aunque sólo a partir de 1950 la provincia como conjunto empieza a tener una inmigración neta. Puesto que la provincia está rodeada de zonas rurales, fuertemente pobladas algunas o con problemas de subempleo, la oleada de inmigración se ha extendido como una ola por las zonas atrasadas, empezando a principios de siglo para los municipios más cercanos y en 1960 para los más alejados.

Por tanto, la industria se inicia en un ambiente de sobrepoblación con fuertes densidades, donde los hijos de las familias campesinas encuentran pocas oportunidades en el ambiente local y se ven forzados a emigrar o bien a buscar trabajo en actividades industriales. Cuando el crecimiento industrial consigue absorber la reserva local de mano de obra, empieza a atraer población de las zonas vecinas. La industria ocupa inicialmente la mano de obra subocupada de las familias campesinas -hijos, mujeres, etc.- que en parte hubiera tenido que emigrar. Aparentemente ocupa el trabajo excedentario de la agricultura sin compartir con ella. Posteriormente, cuando ha alcanzado niveles superiores, empieza a sustituir a los jornaleros del campo y pequeños agricultores y se produce la competencia industria-agricultura por el mercado de mano de obra. En la evolución puede distinguirse una primera etapa en que aumenta la población ocupada en la industria sin disminuir la ocupada en la agricultura, de la segunda en que todos los jornaleros del campo y parte de los propietarios se convierten en industriales y trabajadores de la industria.

Desde el principio la industria ha proporcionado una posibilidad de empleos complementarios a los que no encontraban ocupación suficiente en la agricultura. Los niveles de actividad en el área han debido de ser particularmente altos aun cuando sean especialmente difíciles de medir porque una gran parte de los trabajos se hacen o hacían a domicilio o son trabajos a tiempo parcial que cubren los huecos de la ocupación agrícola. Aun hoy, el nivel de actividad es alto, pues está en un 52,7\% de la población mayor de 16 años que vive en familias 
(más alto que en las provincias vecinas, donde es del 46,2\% en Albacete, del 47,1\% en Murcia y del 48\% en Valencia) (según las E.P.A.). La [211] tasa de actividad de los municipios industrializados es aun mayor, alcanzando valores cercanos al $60 \%$. Las tasas reales son, sin duda, más elevadas porque una parte del trabajo posiblemente no se contabiliza adecuadamente (trabajos complementarios realizados en casa, mujeres que trabajan a domicilio, jubilados, estudiantes, etc.).

Desde el punto de vista del mercado de mano de obra, un aspecto típico de este tipo de industrias ha sido el recurso sistemático al trabajo clandestino y a la ocupación a domicilio. El trabajo negro reviste varias formas y la interpenetración entre trabajo legal e ilegal es muy compleja. Tradicionalmente ha existido un grado de incumplimiento de la legislación vigente incluso en los trabajadores contratados en el sector «formal», pero el trabajo a domicilio ha sido mayoritariamente «negro» y algunos talleres han trabajado completamente «sumergidos». La importancia de la parte «sumergida» de la industria ha variado con el tiempo, tal como puede verse en el siguiente cuadro:

Ocupación en Elda-Petrel-Monóvar en el sector calzado

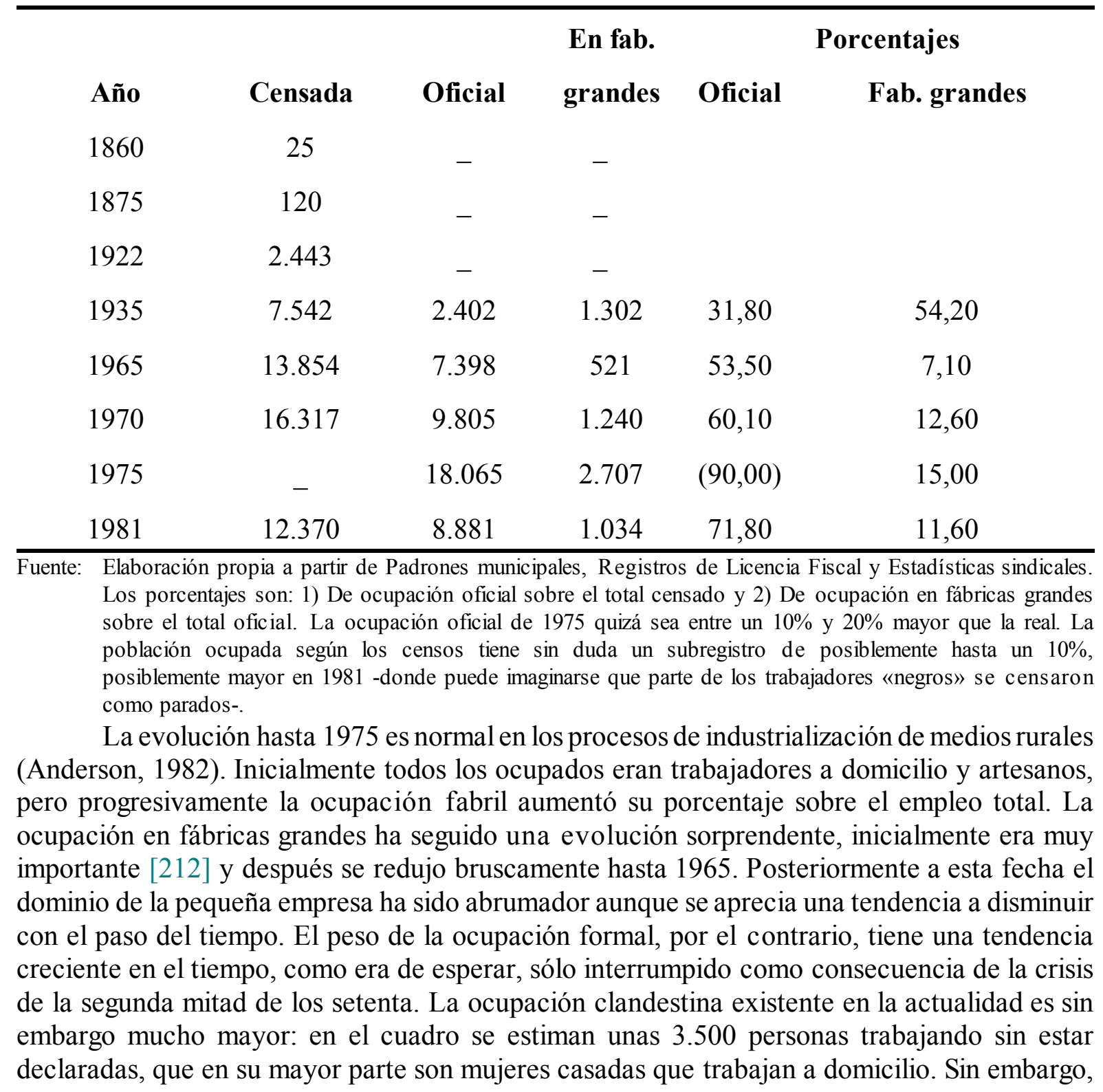


tal como se ve en el siguiente cuadro, en la Comarca hay 5.946 parados que tuvieron un oficio anterior, la mayor parte de ellos zapateros, que presumiblemente están trabajando en gran parte en el sector informal en talleres sumergidos. Si esto es así, posiblemente el sector informal suponga el 50\% de la ocupación total del área.

Ocupación y paro en la comarca Elda-Petrel-Monóvar (1981)

\begin{tabular}{ccccc}
\hline Municipio & Ocupados & Parados (1) & Parados (2) & Total \\
Elda & 14.282 & 1.720 & 3.460 & 19.920 \\
Petrel & 6.129 & 857 & 1.953 & 8.239 \\
Monóvar & 2.968 & 231 & 533 & 3.732 \\
& & & & \\
Total & 23.379 & 2.808 & 5.946 & 31.891 \\
\hline
\end{tabular}

Fuente: Elaboración propia a partir de los padrones municipales.

(1) Parados sin empleo anterior.

(2) Parados con empleo anterior.

La dinámica de la ocupación ha sido muy grande a lo largo del proceso. La provincia, que tiene en la actualidad 1.250.000 habitantes, ha recibido 230.000 personas en los últimos 30 años. El mercado de mano de obra se ha ampliado en 100.000 puestos de trabajo entre 1955 y 1975, a pesar de que la agricultura ha perdido 70.000 puestos, ya que entre la industria y los servicios han compensado el paro rural más los nuevos empleos. Como consecuencia de este trasvase, la agricultura, que contaba en 1955 con el 46\% de la población activa, ha pasado al 11\% en 1980 .

El proceso, sin embargo, ha cambiado desde 1979, pues se han perdido 37.000 puestos de trabajo en 3 años, de los que 17.000 son industriales. Este paro además se ha producido en zonas altamente especializadas donde los [213] trabajadores encuentran pocas oportunidades de empleos alternativos. Como consecuencia, muchos de ellos han pasado a contratarse por debajo de los mínimos acordados en convenio e incluso legales.

En resumen, una ocupación caracterizada por la complementariedad y la sumersión, posiblemente porque esto le proporciona parte de las ventajas comparativas. En la economía de postguerra esto fue un modo de convertir parte de los gastos fijos en variables al igual que ocurre ahora (al menos en parte). Hasta la crisis de la década pasada el sector legalizaba cada vez más las condiciones de ocupación de sus trabajadores, desde entonces la tendencia se ha invertido. La industria dispersa de la zona consiguió ocupar a la fuerza de trabajo excedentaria de la agricultura e incluso paralizar la emigración y atraer a nuevos inmigrantes hasta 1975 aproximadamente. Desde entonces el empleo está estable o disminuye lentamente aunque está aumentando muy rápidamente el trabajo negro.

\subsection{Empresario y capital}

Las primeras iniciativas en el medio rural las tomaron familias sin grandes disponibilidades de capital, aunque tampoco desprovistas de él. Como norma general, no hay diferenciación social en estas primeras etapas entre trabajadores y empresarios, y ambos grupos nacen de los estratos medio y bajos de la estructura social del área. Tuvo cierta importancia la disponibilidad de algunos activos reconvertibles para su utilización a tiempo parcial o completo en la industria, así como las pequeñas acumulaciones financieras locales en manos de 
comerciantes y pequeños agricultores. En el ambiente del umbral de siglo, con un mercado de capital inexistente para estas disponibilidades, aquéllas fueron capturadas por las iniciativas de los empresarios incipientes. Posteriormente, los bancos crearon oficinas en el área, el mercado financiero se regularizó y consecuentemente el papel de estas acumulaciones disminuyó. Con todo, estas aportaciones de capital de riesgo, aunque pequeñas, son decisivas para la introducción de nuevas técnicas que implican renovación o ampliación de activos.

Esta disposición de capital de riesgo no es necesaria en las primeras etapas de la empresa -al menos en las condiciones locales-. Cuando se les pregunta a los empresarios cuál fue la inversión inicial, encuentran natural la respuesta de que la empresa se formó sin ninguna inversión (al menos con una pequeña inversión al alcance de cualquiera). El siguiente inventario proporciona una idea de la financiación de estas pequeñas empresas iniciales:

Esto es consecuencia de la extraordinaria divisibilidad de la tecnología aplicada, a que se aplican varias tecnologías y a que las empresas nacientes utilizan las técnicas más elementales (muchas veces totalmente artesanales) [214]

Inventario de una empresa de calzados de Elda (1914)

\section{Inversión}

\begin{tabular}{llll} 
Disponible & 92,70 & $\begin{array}{l}\text { Exigible a } \\
\text { corto }\end{array}$ & $2.165,25$ \\
Realizable & 459,10 & & \\
Stocks & $1.311,20$ & & \\
Inmovilizado & 721,75 & No exigible & 422,50 \\
\hline
\end{tabular}

Financiación con maquinaria de segunda mano, etc. Sin embargo, en los momentos de rápido crecimiento de la demanda con renovación intensa de los equipos (sobre todo si se acompaña con la introducción de una nueva tecnología) se pueden producir fuertes demandas de capital no satisfechas (como ocurrió p.e. en la década de los años sesenta). A pesar de todo, con frecuencia sólo se utilizan mínimamente las posibilidades reales del mercado de capital, en parte, porque este tipo de empresario suele estar mal informado de su existencia y en parte por la complejidad administrativa de la tramitación y las exigencias de control y garantía de las instituciones financieras. Los hijos de campesinos han tenido la posibilidad de solicitar préstamos con garantía hipotecaria de las tierras, pero los que simplemente han pretendido hacer una pequeña inversión sin garantías reales han encontrado muchos problemas. En este caso tradicionalmente se ha recurrido a la compra de materias primas con operaciones a plazo y el alquiler de la maquinaria más importante. Recientemente la maquinaria se ha comprado a plazos por medio de leasing.

No existe, sin embargo, una historia empresarial acumulativa junto con la formación de capital. Las grandes empresas terminan cerrando sus instalaciones. La historia de la empresa es más bien cíclica: la empresa va creciendo hasta alcanzar un tope, seguidamente se mantiene durante algún tiempo en este nivel para terminar decayendo y cerrando sus instalaciones en un período de crisis general. Presumiblemente esto es debido a que las empresas mayores tienen menos flexibilidad para enfrentarse con la crisis, especialmente porque los costes fijos suponen para ella un porcentaje mayor que para las pequeñas.

Esto implica que la mayor parte de los empresarios de la zona son de primera generación y su experiencia de gestión y cualificación profesional son limitadas. Los cursos que preparan 
algunas instituciones en la ciudad de Alicante para la promoción empresarial parece que tienen una influencia limitada. Fuera del área de estudio, sin embargo, en la ciudad de Alcoy (centro de la industria textil) la experiencia de la Escuela Industrial que prepara técnicos medios ha jugado un papel muy importante en la preparación de técnicos y empresarios que propiciaron la renovación tecnológica de mediados [215] de los años sesenta. La Escuela de Comercio de Alicante y la Universidad pueden ser instituciones muy eficientes para proporcionar una cualificación adecuada a los futuros empresarios si se hacen esfuerzos para adaptar los contenidos académicos a las necesidades locales. En este sentido un centro técnico que imparta la enseñanza especializada relacionada con la industria local -semejante al alcoyano- podría ser un elemento a considerar.

\subsection{Desarrollo de los centros industriales}

Como ya hemos comentado, las primeras industrias nacen en centros bien comunicados, con dimensiones, etc. aunque no sean los más importantes. Aparentemente la modernización de la agricultura crea un conjunto de centros con servicios de gran utilidad para la industria naciente. La industria, sin embargo, no ha aparecido al mismo tiempo en todos los puntos, de modo que la estructura dispersa actual puede verse como consecuencia de dos procesos que han estado actuando en lo que va de siglo:

1. Por una parte la aparición espontánea en algunos municipios de iniciativas empresariales locales dirigidas a la creación de empresas especializadas en productos desconocidos en el área.

2. La difusión de estas primeras iniciativas entre los pueblos vecinos, una vez demostrado que la nueva actividad proporciona rendimientos mayores.

A su vez los núcleos pueden diferenciarse en muy especializados en un sector industrial y poco especializados. En líneas generales los centros del área están muy especializados y tienen poca tendencia a diversificarse con el aumento del tamaño. Al parecer, en los municipios donde la industria se formó a partir de sociedades que combinaban la agricultura de secano de bajos rendimientos con actividades transformadoras hay una elevada especialización local. Es decir, donde la industria aparece por la necesidad o posibilidad de ocupar recursos ociosos para vender a mercados lejanos, pues localmente el mercado es muy limitado. Por otra parte, en las zonas de regadío la industria parece que ha nacido de antiguos artesanos dedicados al suministro de la demanda local (muebles, reparación de maquinaria, alimentaria, etc.) y una vez bien constituida ha abordado los mercados lejanos. Precisamente por ello los centros industriales del regadío tienen una estructura productiva muy diversificada.

En los secanos, una vez especializado el núcleo en un sector industrial, proceso que ha ido unido a una importante corriente migratoria principalmente de los pueblos vecinos, como hemos comentado, la industria empieza a difundirse hacia los centros más cercanos. Este proceso conduce a un crecimiento rápido de la industria de especialización en los nuevos centros, mientras se estabiliza en los antiguos. En las ciudades difusoras empieza a extenderse [216] la ocupación de ramas industriales más próximas (a nivel de tres dígitos de la CNAE) y las industrias que podríamos denominar auxiliares de las principales (artículos para el calzado, maquinaria para el calzado, etc.), crecen los servicios y en general las especialidades o industrias relacionadas que exigen una mayor cualificación profesional y tecnológica, lo cual conduce a un proceso de diversificación creciente. Aun con todo, el nivel de diversificación es pequeño en la actualidad a nivel de dos dígitos de la CNAE. Durante la década pasada esto se ha traducido en una elevada tasa de paro en los municipios centrales mientras que en los marginales la situación es más optimista.

Los centros tradicionales de la industria se están comportando, por tanto, como centros 
de servicios que suministran a los vecinos y, como consecuencia, el peso del sector servicios se ha desarrollado considerablemente en la década pasada. Sin embargo, el incremento del sector servicios y de las industrias auxiliares no alcanza a generar un empleo suficiente -e incluso puede afirmarse que hoy este sector es insuficiente en muchos aspectos para las necesidades locales-. En consecuencia, la repercusión de las crisis sectoriales es grave en estos municipios. Pero, puesto que la situación parece madura para promocionar el desarrollo de industria auxiliar (maquinaria, productos químicos, servicios de asistencia, etc.) hemos de pensar que una política de promoción de servicios podría tener éxito y, en cualquier caso, se hace necesario promover la diversificación de los antiguos núcleos desarrollando industrias más intensivas en trabajo cualificado y tecnología. La estructura industrial formada por industrias muy pequeñas dirigidas por empresarios con niveles de cualificación relativamente bajos y descapitalizados por la crisis es un cuello de botella para avanzar en esta dirección.

\section{BIBLIOGRAFÍA}

ALCAIDE INCHAUSTI, J., Contabilidad de la Región Valenciana, Valencia, Consejo Económico Social Sindical de la Región Valenciana, 197 pp.

ANDERSON, D., «Small industry in Developing Countries: A discusion of Issues», World Development, vol. 10, núm. 11 (1982), pp. 913-948.

ANDERSON, et alt., La empresa rural y el empleo no agrícola, Banco Mundial, Washington, 1978, $95 \mathrm{pp}$.

ARACIL, R. y GARCÍA BONAFE, M., Industrialització al País Valencià (el cas d'Alcoi), Valencia, 3 i 4, 1974, 315 pp.

BELANDO CARBONELL, R., Estudio demográfico de Monóvar (ss. XVI-XX), Universidad de Alicante, 1980, 192 pp. [217]

BERNABÉ MAESTRE, J. M., Industria i subdesenvolupament al País Valencià, Mallorca, Ed. Moll, 1975, 109 pp.

BERNABÉ MAESTRE, J. M., La industria del calzado en el Valle del Vinalopó, Dto. de Geografía, Universidad de Valencia, 1976, 236 pp.

BERNABÉ MAESTRE, J. M., «Factores de localización y crisis de la industria del calzado», Panorama bursátil, año II, 1977, núm. 6, pp. 71-87.

-«Industria y movimientos de población», Ciudad e industria, Oviedo, 1977, pp. 54-65.

BROWN, L. A., Innovation Diffusion. A New perspective, Methuen, London, 1981, 345 pp.

CASADO SÁNCHEZ, M. A., Ibi. Estudio geoeconómico de un núcleo industrial, Dto. de Geografía, Universidad de Valencia, 1974, 93 pp.

CHENERY, H. B. y KEESING, D. B., The Changing Composition of Developing country exports, World Bank Staff Working Paper No. 314, 1979 (mimeografiado), 50 pp.

CLAVER, E. et alt., Plan de estabilización e industrialización de la economía alicantina, Alicante, Dip. Provincial, 1982, 335 pp.

COOPER, Ch., Policy Interventions for Technological Innovation in Developing Countries, World Bank Staff Working Paper N. ${ }^{\circ}$ 441, 1980 (mimeografiado), 59 pp.

COUNCIL OF EUROPE, Mobilising the indigenous potential of disavantaged regions. A new dimension of regional planning, Strasbourg, 1982, mimeografiado, 38 pp.

CRESPO GINER, J., San Juan. Estudio demográfico-económico de un municipio de la huerta de Alicante. Instituto de Estudios Alicantinos, 1979, 113 pp.

DEFFONTAINES, P., «Los horizontes de trabajo en el macizo de Alcoy», Estudios Geográficos, núm. 71, 1958, pp. 275-280.

MURIES, Tablas input-output de la Provincia de Alicante, Alicante, Dip. Provincial, 1979, 160 pp. 
FUA, G., Occupazione e capacità produttive: la realtà italiana, Bologna, II Mulino, 1975, 123 pp.

FUA, G. y ZACCHIA, C. (ed.), Industrializzazione senza fratture, Bologna, II Mulino, 1983, $334 \mathrm{pp}$.

GIL OLCINA, A., «El regadío de Elche», Estudios Geográficos, n. ${ }^{\circ} 112-113,1968$, pp. 527-571. -La propiedad señorial en tierras valencianas, Del Cenia al Segura, Valencia, 1979, 276 pp.

GOZÁLVEZ PÉREZ, V., «Notas sobre demografía de la provincia de Alicante», Cuadernos de Geografía, núm. II, Valencia, 1972, pp. 149-199.

-La industria de esteras y alfombras en Crevillente, Alicante, Círculo de Economía, 1975, 41 pp. [218]

-La ciudad de Elche. Estudio geográfico, Dto. de Geografía, Universidad de Valencia, 1976, 290 pp.

-El Bajo Vinalopó. Geografía agraria, Dto. de Geografía, Universidad de Valencia, 1977, 270 pp.

INESCOOP, Boletín del servicio de estudios, años 1974 a 1983.

IBARRA PÉREZ, J. A., Les precondicions per al desenvolupament economic de les comarques meridionals del País Valencià, Alicante, Caja de Ahorros Provincial de Alicante, 1978, 99 pp. JORDÀ BORRELL, R. M., «La industria del turrón en Jijona», Cuadernos de Geografía, 1973, n. ${ }^{\circ} 13$, pp. $57-74$.

LEE E.(ed.), Export-led industrialisation and development, I.L.O. Asian employment program, Ginebra, 1981, 206 pp.

LÓPEZ GÓMEZ, A., ROSSELlÓ VERGER, V. M. et al., Geografía de la provincia de Alicante, Diputación Provincial de Alicante, 1978, 615 pp.

-Geografia de les terres valencianes, Valencia, 3 i 4, 1977, 263 pp.

-«El puerto de Alicante», Estudios Geográficos, 1955, n. ${ }^{\circ}$ 60, pp. 511-584.

KORTEN, D., Community organization and rural development: A Learning Process Approach, Ford Foundation, New York, 1983, 32 pp.

MORALES GIL, A., El Altiplano de Jumilla-Yecla, Universidad de Murcia, 1972, 467 pp.

O.C.D.E., Les capacites d'entrependre en milieu rural, Compte rendu..., mimeografiado, París, 1983, $18 \mathrm{pp}$.

O.C.D.E., Cooperative action programme. Rural entrepreneurial capacities. Provisional programme, mimeografiado, Working document, París, 1983, 12 pp.

O.C.D.E., «The role of regional policy in suporting the mobilisation of indigenous potential», en Rural entrepreneurial capacities, Working document, mimeografiado, París, 1983, 6 pp.

ONUDI, Industrialización y desarrollo rural, New York, O.N.U., 1979, 115 pp.

PÉREZ PUCHAL, P., «Las densidades demográficas en la región valenciana», Estudios geográficos, n. ${ }^{\circ} 112-113$, pp. 475-498.

-Geografia de la població valenciana, València, L'Estel, 1976, 170 pp.

PIQUERAS, J., La vid y el vino en el País Valenciano, Valencia, Diputación, 1981, 344 pp.

PREVASA, Informaciones-1977, Caja de Ahorros de Valencia, 1978.

-La estructura de la producción en el País Valenciano, en Estudios Básicos para la Ordenación del Territorio en el País Valenciano, vol. III, 497 pp.

QUEREDA SALA, J. J., Comarca de la Marina (Estudio de Geografía Regional), Diputación Provincial de Alicante, 1978, 431 pp.

RAPOSO SANTOS, J. M., et alt., Situación actual y perspectivas de desarrollo de la Región Valenciana, CECA, Madrid, 1976, 4 vols. [219]

RICO, A. et alt., L'economia del País Valencià. Estratègies sectorials, Valencia, Inst. Alfonso el Magnánimo, 1982, 2 vols.

ROSSELLÓ VERGER, V. M., «Ensayo de una división comarcal en la Provincia de Alicante», 
Cuadernos de Geografía, n. ${ }^{\circ} 1,1964$.

-(Dir.), Estudio socio-económico de Elche y su comarca, mecanografiado, Valencia, 1979, 3 vols.

STEED, G. P. F., «Product differentiation, location protection and economic integration: western europe's clothing industries», Geoforum, vol. 9, 1978, pp. 307-318.

TAAFE, E. J. et alt., «Transport expansion in underdeveloped countries: a comparative analisis», Geographical Review, n. ${ }^{\circ} 53$ (1963) pp. 503-529.

VÁZQUEZ, A., Industrialización espontánea, mimeografiado, Univ. Autónoma de Madrid, 1982.

-Las pequeñas y medianas empresas en España: Problemas y políticas, 1982.

-«National contribution. Spain», en O.C.D.E., Working Document, Cooperative action programme, rural entreprenueurial capacities, París, 1983. 